\title{
RTDF2009-18030
}

\section{STRUCTURAL CRASHWORTHINESS STANDARDS COMPARISON: GRADE-CROSSING COLLISION SCENARIOS}

\author{
Patricia G. Llana \\ U.S. Department of Transportation \\ Volpe Center \\ Cambridge, Massachusetts, USA
}

\begin{abstract}
In support of the Federal Railroad Administration's (FRA) Railroad Equipment Safety Program, American and European grade-crossing collision scenarios were evaluated and compared. Finite element analyses (FEA) were employed to subject an FRA-compliant passenger car to grade-crossing collision scenarios defined in both the proposed FRA Code of Federal Regulations (CFR) and European Standard (EN) 15227. The proposed FRA collision scenario involved a single car impacted by a cart. The cart had a punch mounted to it to hit a specific post of the end frame of the car. The EN 15227 collision scenario involved a complete train consist impacting a large deformable obstacle that approximates a lorry. The analyses show that these collision scenarios, while both gradecrossing scenarios, are very different not only in terms of the impact object and the amount of initial kinetic energy involved, but also in terms of how the car is loaded and deformed during impact. The FRA scenario is shown to be easier to analyze as well as easier to test than the EN 15227 scenario. Additionally, the FRA scenario is safer to test because of the levels of initial energy involved. The FRA scenario also provides clearer metrics of success. The FRA-compliant car utilized in the analyses and test conducted for this paper passed both FRA and EN 15227 grade-crossing collision scenarios according to the requirements for each respective standard. However, the analyses show that despite both scenarios providing for energy absorption in a grade-crossing collision, because the manner in which the car is loaded and deformed (concentrated vs. distributed) is different, the FRA performance standard and EN 15227 grade-crossing collision scenarios are not equivalent and mutual compliance is not guaranteed.
\end{abstract}

\section{INTRODUCTION}

Proposals have been submitted to FRA to operate equipment built to European standards EN 12663 and EN 15227 in the U.S. [1]. EN 12663 is a long-standing standard that specifies design requirements for passenger equipment, such as occupant volume strength and static loading conditions. EN 15227 is a more recent standard that specifies performance requirements. It dictates crash energy management (CEM) requirements meant to enhance the crashworthiness of passenger equipment. The intent of both standards is to help ensure the passengers and crew have a safe volume within which to ride out a collision or derailment. In other words, the occupied volume is preserved with no intrusion of car structure or foreign objects.

Due to differences in operating environments and loading conditions, the two standards are sufficiently different that comparison is difficult. The pertinent structural concerns are in the following areas:

- Grade-crossing collisions,

- Train-to-train collisions,

- Occupant volume strength, and

- Anticlimbing, truck attachment strength, side strength, and rollover strength.

This paper addresses grade-crossing collisions by comparing a grade-crossing collision scenario from the CFR to a grade-crossing collision scenario from EN 15227. The FRA performance standard and the European performance standard will be evaluated by comparing the outcomes of their respective grade-crossing collision scenarios.

The Volpe National Transportation Systems Center (Volpe Center) and FRA have been engaged in active research aimed at improving rail vehicle crashworthiness. The objective of the research performed is to incrementally improve safety. Full-

This material is declared a work of the U.S. Government and is not subject to copyright protection in the United States. Approved for public release; distribution is limited. 
scale crashworthiness research has focused on two areas: grade-crossing collisions and train-to-train collisions. Each is characterized by a collision scenario. Scenarios are developed to bound the range of potential threats as opposed to recreating a single accident condition. In the train-to-train collision scenario, a leading cab car hits another train, head on. In this scenario, both the underframe and the superstructure of the cab car are loaded. In the grade-crossing collision scenario, a leading cab car hits an object at a grade crossing such that only the superstructure is loaded and the underframe is not. Because the underframe of a railcar provides the majority of the strength to protect the passengers, this collision scenario can be dangerous for operators and passengers alike if the end structure is breached by the colliding object. Photographs from two such accidents at grade crossings in the U.S. are shown in Figure 1. In the photographs, either a collision post or corner post has been severed by the colliding object. In the Portage, Indiana accident shown in the left photograph of Figure 1, the object was a 6-foot diameter, 19-ton coil of sheet steel that punctured the end of the car, severing the collision post, and traveled half the length of the car, killing three people [2]. In the Milford, Connecticut accident shown in the right photograph, a truck collided with the cab car, severing the corner post.
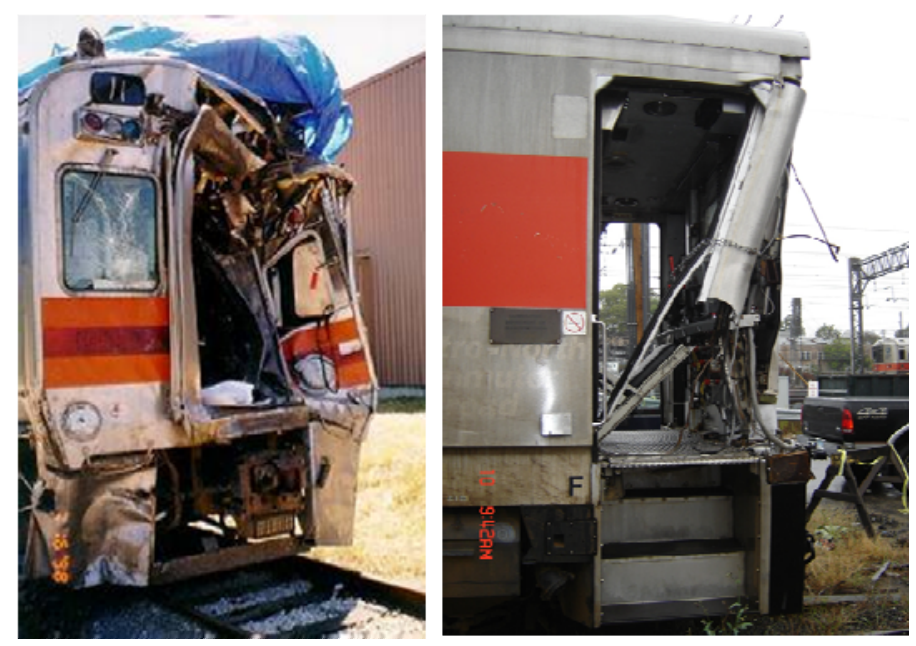

Figure 1. Grade-Crossing Accidents that Have Involved Focused Load on the Superstructure

New FRA standards have been proposed to address a range of potential threats where the superstructure is loaded in a concentrated manner as opposed to across the full front end. The new standard is intended to increase the strength requirements for cab car end structures and to impose additional requirements for energy absorption and maximum allowable intrusion during a collision [3]. FRA has issued a notice of proposed rulemaking (NPRM) to include these new standards in 49CFR238. By encouraging improved energy absorption capabilities for end structures, the rule aims to improve survivability for operators and passengers at incrementally higher collision speeds. The intent of the new FRA standard is to:

1. Limit intrusion into the occupant volume,

2. Ensure energy absorption by deforming substantially before failure of structural components occurs,

3. Promote an integrated end frame by shedding the impact load into the underframe and roof structures,

4. Facilitate demonstrable compliance, which is verifiable either by analysis or test, and

5. Allow applicability to a range of equipment.

\section{FRA-Compliant End Frame Design}

The state-of-the-art (SOA) end frame design was developed specifically for a series of tests conducted in support of the new FRA standard. The SOA design includes improvements over older designs that are intended to increase the energy absorption during a collision and provide a survivable space for the operator and passengers. The passenger car used in the tests conducted in support of the new FRA standard was a Budd M1 passenger car retrofitted with an SOA end frame structure. The SOA end frame, shown in Figure 2 retrofitted to a Budd M1 car, includes more substantial collision and corner posts, more robust post connections to the buffer beam and anti-telescoping (AT) beam, shelves and bulkhead sheets that are integrated with the collision and corner posts, and stronger end frame support with full-length side sills [5][6].

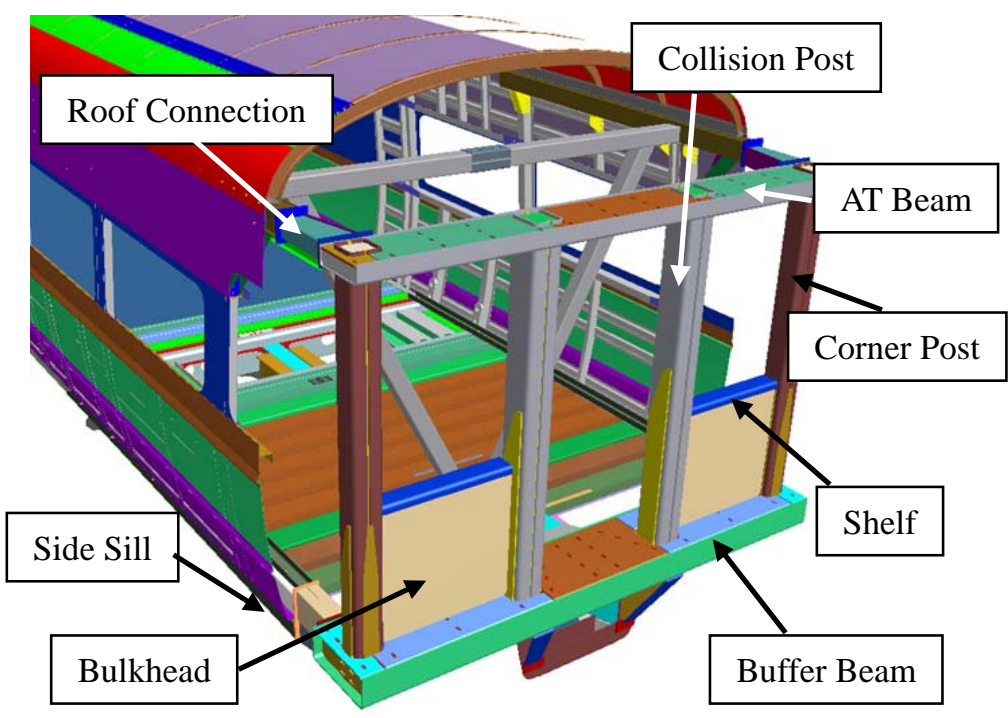

Figure 2. Schematic of the SOA End Frame 


\section{GRADE-CROSSING COLLISION SCENARIOS}

The steel coil impact is an exemplar of the type of threat that the grade-crossing collision scenario in the new FRA standard is meant to address. In this scenario, an object impacts either a corner post or a collision post of the end structure of the car. Figure 3 provides a schematic depiction of the gradecrossing collision scenario found in the FRA standard. In this scenario, the stationary car must withstand a frontal impact with a moving rigid object. The rigid object has a striking surface mounted to it such that it strikes the front end structure at either a single corner or collision post. In this scenario, only one post is impacted, either one collision post or one corner post. For this paper, the collision post impact will be evaluated.

The grade-crossing collision scenario in EN 15227 that most closely resembles the FRA standard collision scenario is a train unit front end impact with a large deformable obstacle on a level crossing (design collision scenario \#3) [4]. Figure 4 provides a schematic depiction of the EN 15227 grade-crossing collision scenario. In this scenario, a complete train unit impacts a stationary, large deformable obstacle that resembles a lorry.

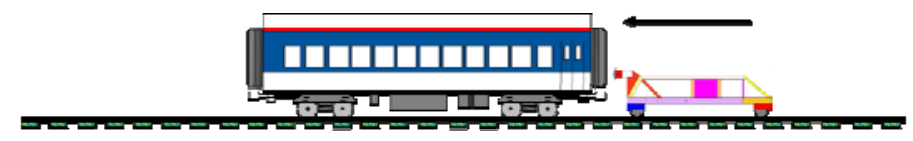

Figure 3. FRA Grade-Crossing Collision Scenario

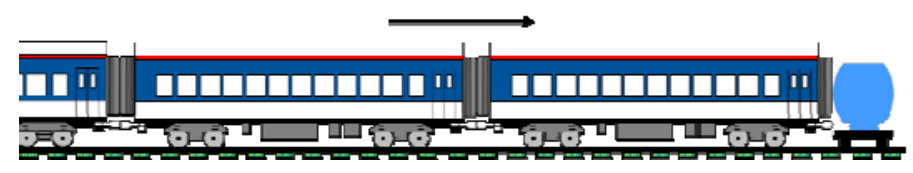

Figure 4. EN 15227 Grade-Crossing Collision Scenario

An initial comparison of these grade-crossing collision scenarios reveals marked differences. The FRA scenario involves one stationary passenger car hit by a rigid impact object, resulting in the load being focused on the superstructure, specifically, one collision post. The EN 15227 scenario involves a complete train consist hitting a large deformable obstacle, resulting in the load being distributed over the entire end structure of the first car of the consist. Another marked difference between the FRA standard and EN 15227 is that the FRA performance requirement can be satisfied by either analysis or test, whereas the EN 15227 performance requirement can only be satisfied by analysis because there are no lorries in reality that provide the characteristics required in the standard. In this paper, results from both a full-scale dynamic test and finite element analysis are provided for the FRA grade-crossing scenario, whereas only finite element analysis results will be provided for the EN 15227 gradecrossing scenario.
The FRA performance standard and the European performance standard will be evaluated by comparing the outcomes of their respective grade-crossing collision scenarios. The same passenger car and end frame structure (M1 Budd car retrofitted with an SOA end frame) will be used to evaluate each standard; however the results will be verified according to the standard being evaluated.

\section{FRA PERFORMANCE STANDARD}

The objective of the FRA performance standard is to ensure an end structure that provides operator and passenger protection in a collision in which only the superstructure is impacted. The end structure should be designed to deform gracefully and absorb a minimum amount of energy. Specifically, the end frame and supporting car body structure must be capable of absorbing a minimum of 135 foot-kips (ftkip) of energy. There shall be no more than 10 inches (in) of longitudinal permanent deformation into the occupied volume as a result of the collision, with no complete post failure. The intent of this requirement is to preserve the space in the operator's compartment.

The schematic for both the full-scale dynamic test and the FEA analysis performed is shown in Figure 5. In the full-scale test and the FEA, a single car (Budd M1 retrofitted with the SOA end frame) is impacted by a rigid cart. The cart has a punch mounted to the front that loads one collision post of the end frame approximately 30 in above the underframe. The punch on the cart has a 4-foot diameter to mimic a steel coil (indicated by the dashed blue circle in the figure). The nominal weights of the car and the impact car, and the speed of the cart were adjusted to impart the $135 \mathrm{ft}$-kip minimum energy required. In this case, the car weighed approximately 80 kips, and the cart weighed approximately 14 kips and traveled at approximately 19 miles per hour (mph). In the FEA, a halfsymmetric model was utilized to take advantage of geometrical symmetries and more efficiently utilize computational analysis time.

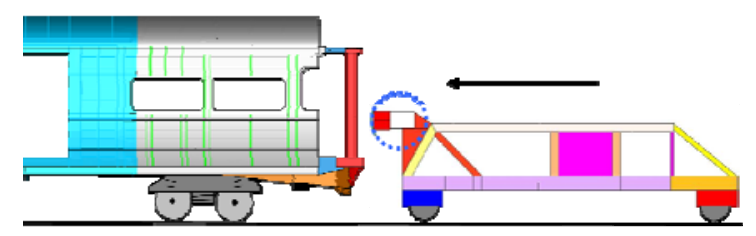

Figure 5. FRA Grade-Crossing Dynamic Test Schematic

\section{FRA Performance Standard: Collision Post Dynamic Test Results}

Figure 6 shows still frames of the test taken from the highspeed video. The top photo shows the car and cart at the moment of impact. The middle photo shows the collision post at its maximum deflection. The bottom photo shows the car and 
cart at the end of the test, after some elastic energy has been recovered from the collision post [7].
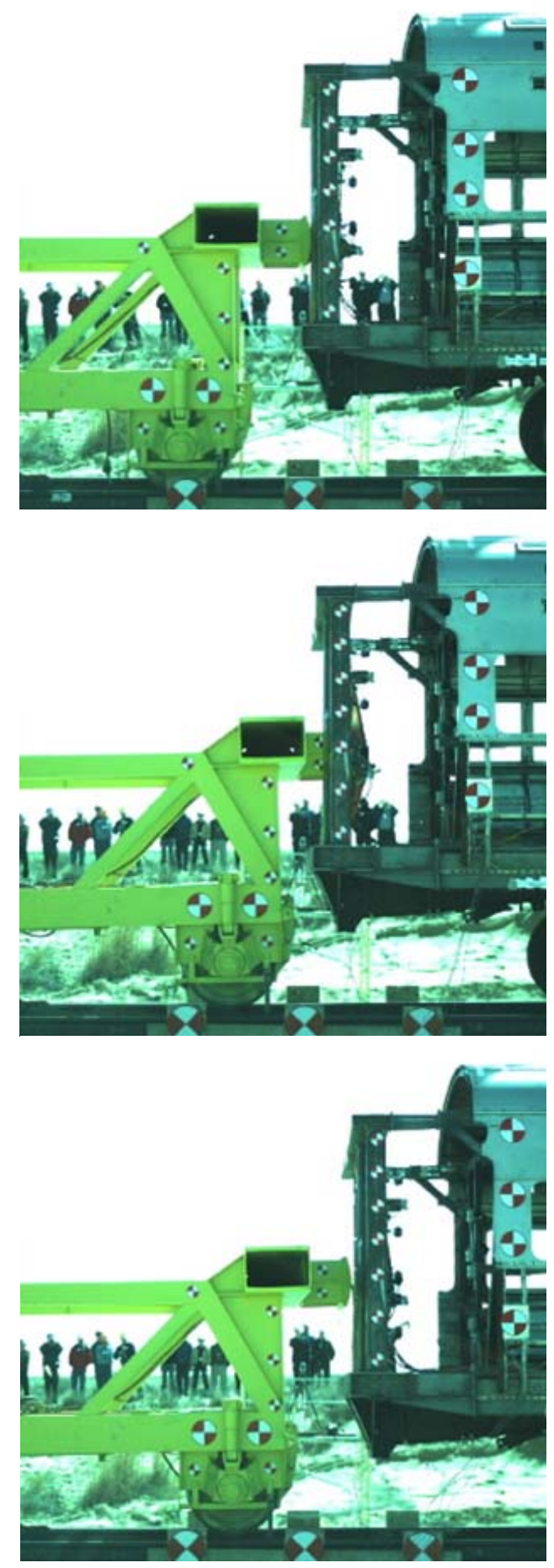

Figure 6. Still Photographs from High-Speed Videos

A photograph of the end frame taken after the test is shown in Figure 7. The permanent deformation of the collision post was approximately 7.4 inches, meeting the requirement that there be less than 10 inches of crush. The end frame absorbed approximately $138 \mathrm{ft}$-kips of energy, exceeding the minimum energy absorption requirement of $135 \mathrm{ft}$-kips. Some fracture of the collision post occurred behind the point of impact as well as at the connection to the buffer beam, but there was no complete collision post failure. Therefore, all of the requirements of the FRA standard were met by this full-scale dynamic test.

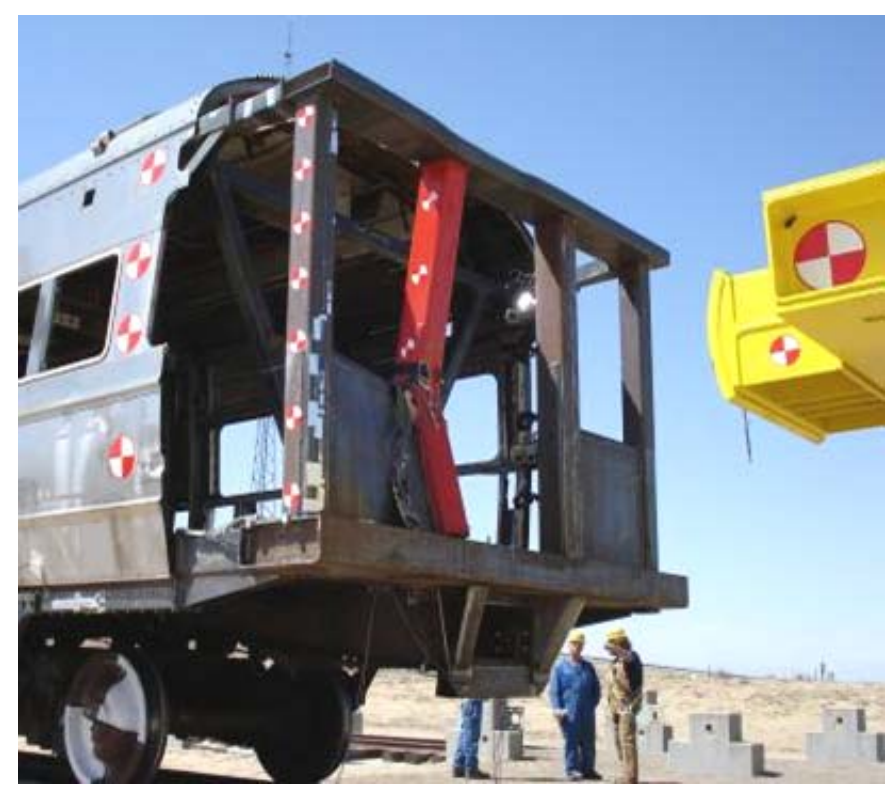

Figure 7. The End Frame after the Dynamic Test

\section{FRA Performance Standard: FEA Results}

As a result of the FEA performed, the permanent deformation of the collision post was approximately 7 inches, meeting the requirement that there be less than 10 inches of crush. The end frame absorbed approximately $135 \mathrm{ft}$-kips of energy, meeting the minimum energy absorption requirement of $135 \mathrm{ft}$-kips. There was some fracture of the collision post behind the point of impact as well as at the connection to the buffer beam, but there was no complete collision post failure. As with the dynamic test results, all of the requirements of the FRA standard were met by the FEA results. The fracture at the base of the collision post from the full-scale dynamic test is shown in Figure 8, and the fracture predicted by the FEA results is shown in Figure 9. Therefore, not only were the requirements of the FRA standard met, but the FEA results are in good agreement with the full-scale dynamic test results. 


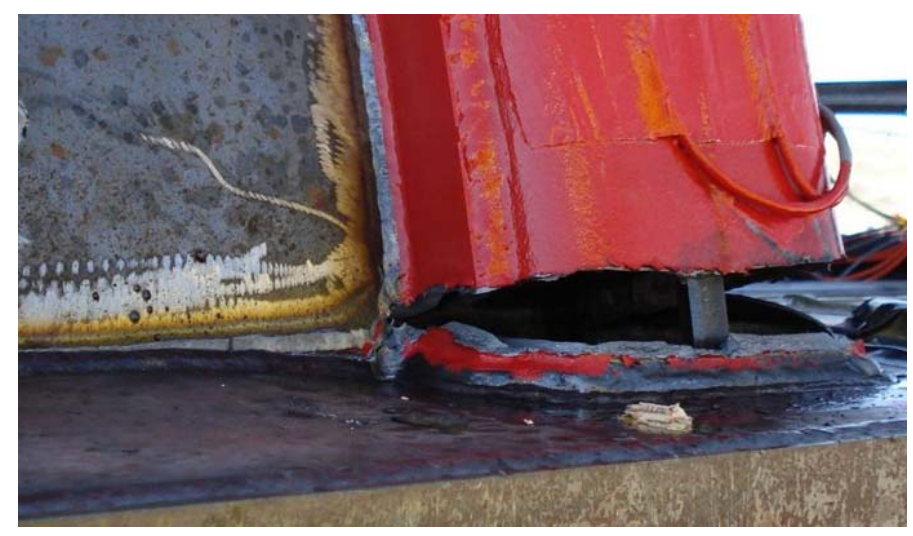

Figure 8. Post-Test Photograph of the Base of the Collision Post Following Cart Impact

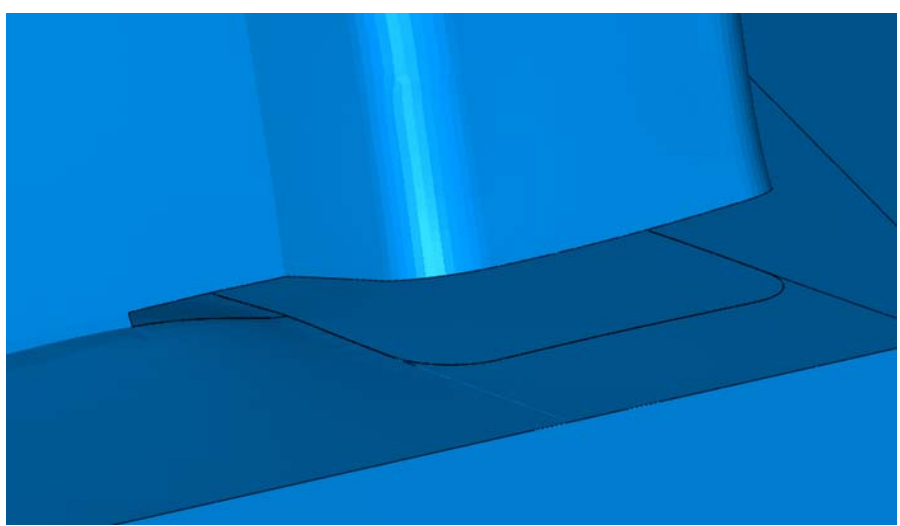

Figure 9. Deformation at the Base of the Collision Post Predicted by FEA

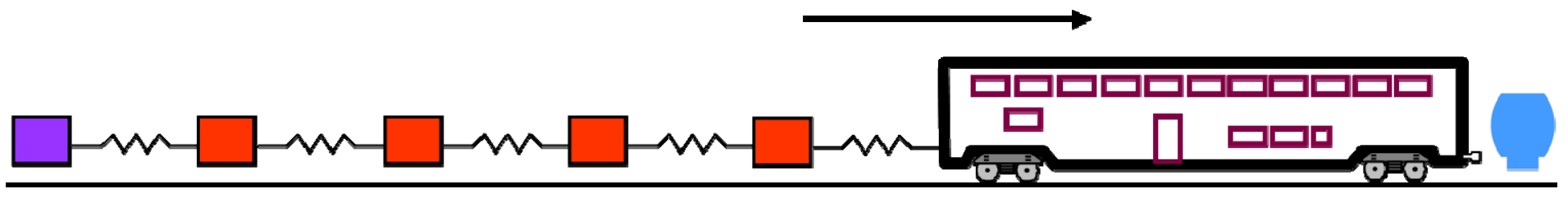

Figure 10. EN 15227 Grade-Crossing Schematic of Collision Scenario \#3

\section{EN 15227 PERFORMANCE STANDARD}

The objective of the EN 15227 performance standard is to provide a CEM standard that complements the EN 12663 design standard. EN 15227 dictates that passenger cars be designed to absorb collision energy in a controlled manner, provide operator and passenger protection in a collision, and reduce the risk of override and derailment.

EN 15227 requires that the structural integrity and survival space of the occupied areas must be maintained and that the mean deceleration must be less than $7.5 \mathrm{~g}$. The schematic for the EN 15227 Impact scenario \#3 FEA is shown in Figure 10. In the 3D FEA model, a complete train unit impacts a large deformable obstacle. The speed of the train was $53 \mathrm{mph}^{1}$.

The complete train unit consists of a leading cab car, 4 trailing coach cars (shown in red), and a locomotive (shown in purple). In the analysis, only the leading cab car is modeled explicitly, the rest of the cars are modeled as masses connected by springs. The springs are defined to represent the coupled connections between the cars. The leading cab car and trailing coach cars weighed approximately 100 kips each, and the trailing locomotive weighed approximately 267 kips.

The large deformable obstacle in the analysis is meant to represent a lorry. The lorry has a mass of 15,000 kg (33 kips), a

${ }^{1}$ This speed was chosen as the same speed used in the FEA conducted by Stadler Rail Group for their GTW DMU developed for Capital Metro in Austin, Texas [1]. center of mass $1750 \mathrm{~mm}$ (69 in) above rail, and uniform axial density and stiffness. The dimensions of the lorry are shown in Figure 11.

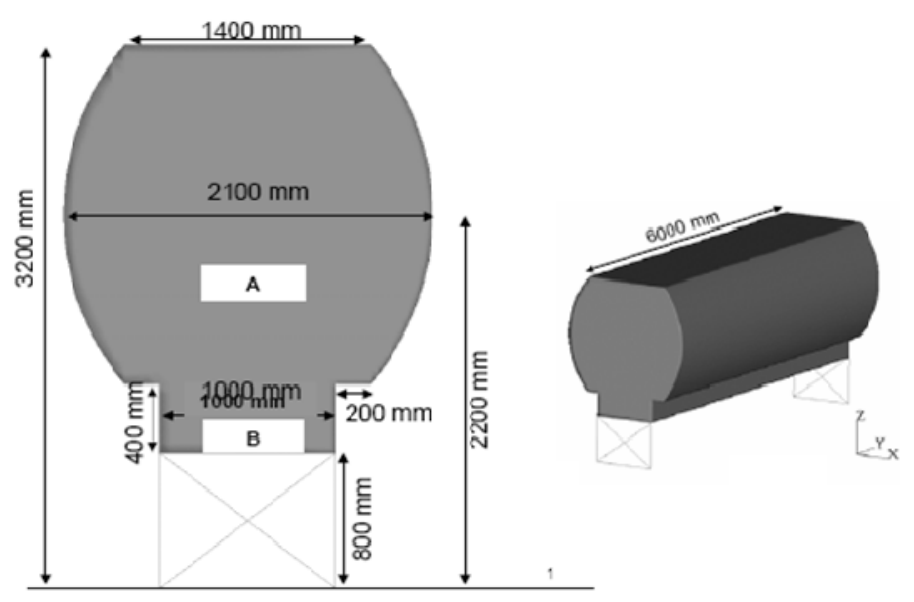

Figure 11. Large Deformable Obstacle Shape and Dimensions [4]

The stiffness of the lorry must be such that the characteristics of its longitudinal force-displacement behavior are at least those given by EN 15227, shown in Figure 12. The 
lorry force-displacement behavior was determined from an FEA of the impact of the lorry with a rigid sphere of mass $50,000 \mathrm{~kg}$ (110 kips) traveling $30 \mathrm{~m} / \mathrm{s}$ (67 mph), as shown in Figure 13. In Figure 12, the lorry force-displacement behavior is shown in red and increases rapidly after 20 in of crush. As will be shown later, the maximum crush of the lorry in the grade-crossing impact analysis is approximately 9 in; therefore, the rapid increase in the behavior exhibited after 20 in is not a concern for this analysis.

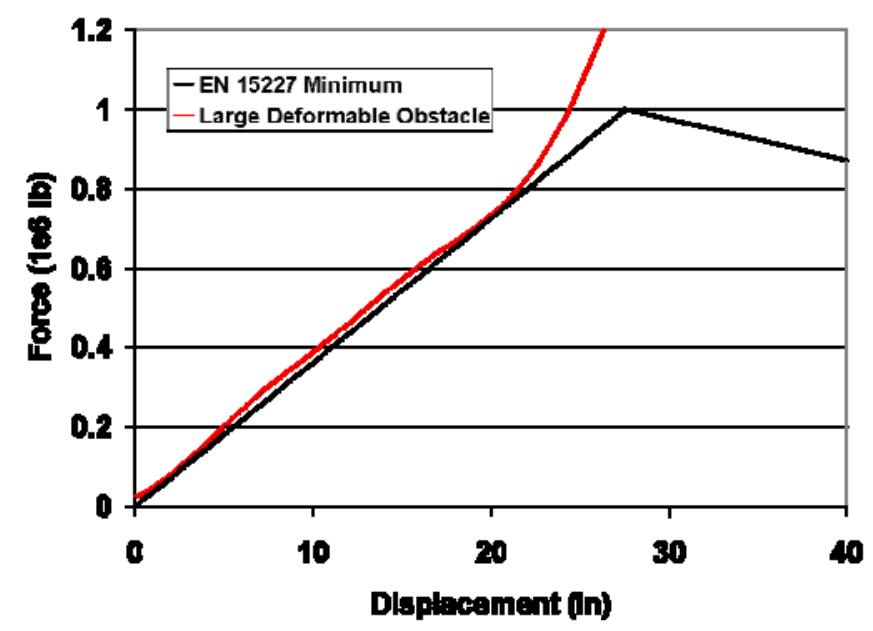

Figure 12. Large Deformable Obstacle Force-Displacement Behavior

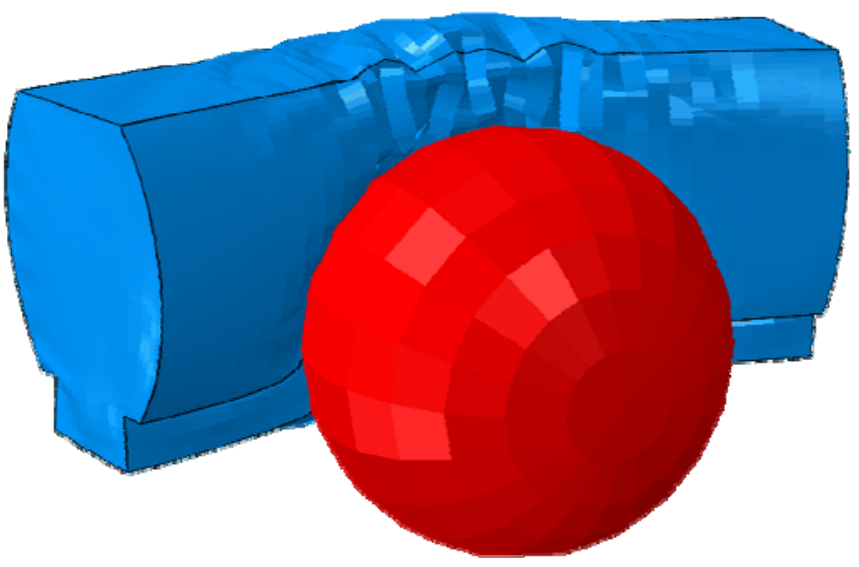

Figure 13. Large Deformable Obstacle Impacted by Rigid Sphere

The complete FEA model for the EN 15227 impact scenario \#3 is shown in Figure 14. The model shows the lorry on the right with the leading cab car explicitly modeled and the trailing coach cars and locomotive modeled as masses and springs. Figure 15 is a close-up of the leading cab car and lorry.

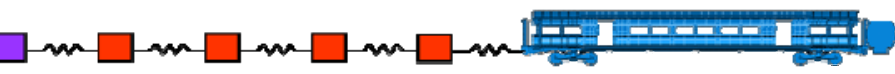
Figure 14. Complete FEA Model for EN 15227 Grade- Crossing Collision Scenario \#3

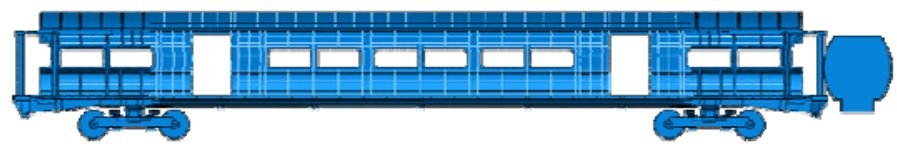

Figure 15. FEA Model of First Car and Lorry

\section{EN 15227 Performance Standard: FEA Results}

The resulting longitudinal vehicle velocities after impact are shown in Figure 16. The lorry velocity is black, and the leading cab car velocity is red. The train consist starts the analysis at $53 \mathrm{mph}$, and the lorry is stationary. After initial impact, the cars in the consist slow down and then speed up again as the springs react to the impact. The lorry speeds up, is pushed by the end frame, then is projected forward by the stillmoving consist. Because the mass of the lorry is much less than the mass of the consist and much of the energy is elastic, at the end of the analysis the lorry travels at a speed faster than the initial speed of the consist. A large amount of energy is involved in the impact compared with how much energy is dissipated through deformation of the cab car end structures and lorry. At the end of the analysis, the last spring in front of the trailing locomotive is still loaded, as shown in light blue in Figure 16.

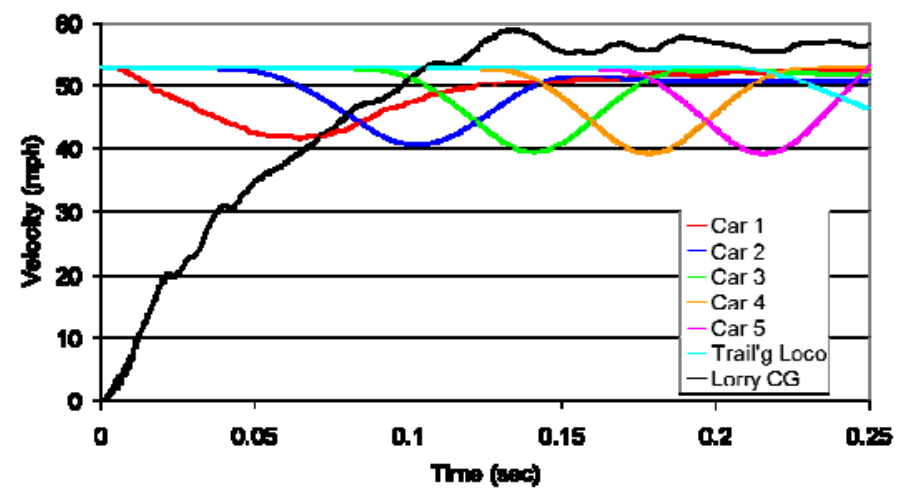

Figure 16. Longitudinal Vehicle Velocities after Impact

Figure 17 shows the deformed shape of the end frame structure on the leading cab car, and Figure 18 shows the 
deformed lorry. Almost all of the deformation occurred in the end frame, the end frame supporting structure, and draft sill behind the end frame. Very little deformation occurred in the carbody.

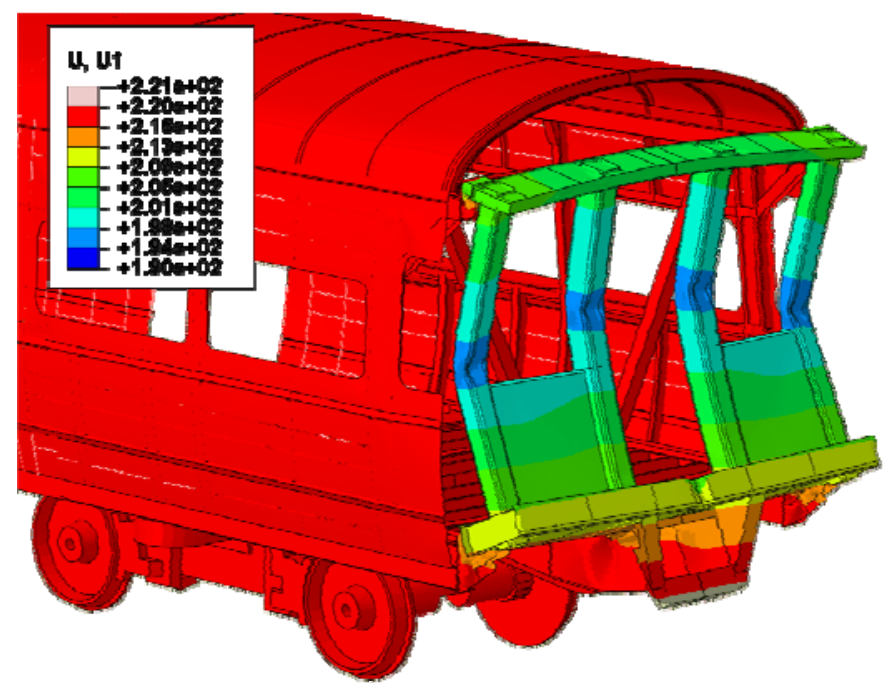

Figure 17. Deformed End Frame and Supporting Structure on Leading Cab Car

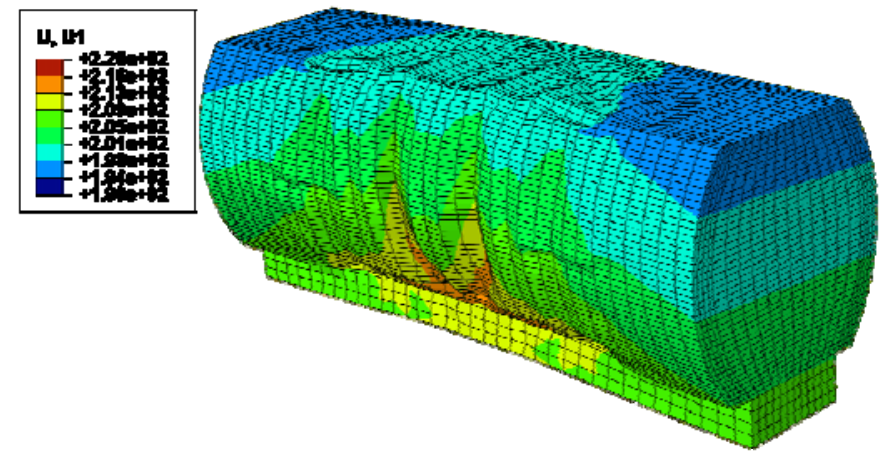

Figure 18. Deformed Lorry with Indentations from Cab Car End Frame

As a result of the impact, the end frame has conformed to the lorry shape. Similarly, the dents from the collision posts, corner posts, shelves, AT beam, and buffer beam are evident in the lorry. The collision and corner posts have bent around the lorry. However, they are bent at different vertical heights. The AT beam and the buffer beam have also deformed in bow like shapes around the lorry. This cab car has no CEM design features but the supporting structural members behind the end frame act like energy-absorbing elements. The SOA end frame works the way it is supposed to work, with the end frame acting in an integrated manner and the posts bending and absorbing energy.

The following results show the energy absorbed by the train at different levels: at the train level, at the car level, and at the end frame level. Figure 19 shows the strain energy present in the consist and the lorry at the end of the analysis. The springs between the passenger cars have all unloaded; therefore, no strain energy is left in those springs (Connections 1 through 4 in Figure 19). As stated previously, the spring preceding the trailing locomotive (Connection 5 in Figure 19) has not had a chance to unload like the other springs; therefore, strain energy is still stored in the spring, which is all recoverable energy. As a result of the impact, energy is dissipated only by the deformation of the end structure of the leading cab car (Car 1) and the lorry. Approximately 1,370 ftkips of energy is absorbed by the end structure of the leading cab car, and approximately $950 \mathrm{ft}$-kips of energy is absorbed by the lorry.

Figure 20 shows the energy absorbed at the car level, more specifically the energy dissipated in Car 1 . Figure 20 separates the strain energy absorbed by the end frame (shown in red), the end frame to carbody connections (shown in yellow), and the remainder of the carbody (shown in blue). As is shown in the figure, approximately one-half of the energy absorbed by Car 1 is absorbed by the structural members connecting the end frame to the carbody, approximately one-third is absorbed by the end frame, and approximately one-sixth is absorbed by the remainder of the carbody. The EN 15227 impact scenario effectively turns the connecting structural members into a CEM system. A lot of energy is absorbed by components that are not designed to absorb energy. And an even larger amount of energy is being dissipated by the connecting members than by the end frame itself.

Looking at the energy dissipated at the end frame level, Figure 21 shows the strain energy absorbed by the components of the end frame. The figure shows the amount of energy absorbed by one of the collision posts, one of the corner posts, and by the AT beam and end beam combined as a result of the collision. The collision post is highlighted in orange, and the corner post in green. For the EN 15227 collision scenario, the collision post and the corner post absorb almost equal amounts of energy, and the AT beam and end beam absorb more energy than the collision post and corner post combined.

Included in Figure 21 are the results from the FRA standard FEA results for the collision post impact. This figure shows the very different distributions of energy absorption in the end frame between the EN 15227 and the FRA collision scenarios. For the FRA collision scenario for impact into one collision post, the collision post absorbs most of the energy, with the corner post, AT beam, and end beam absorbing very little energy. Also, the collision post absorbs more energy in the FRA scenario than in the EN scenario. 


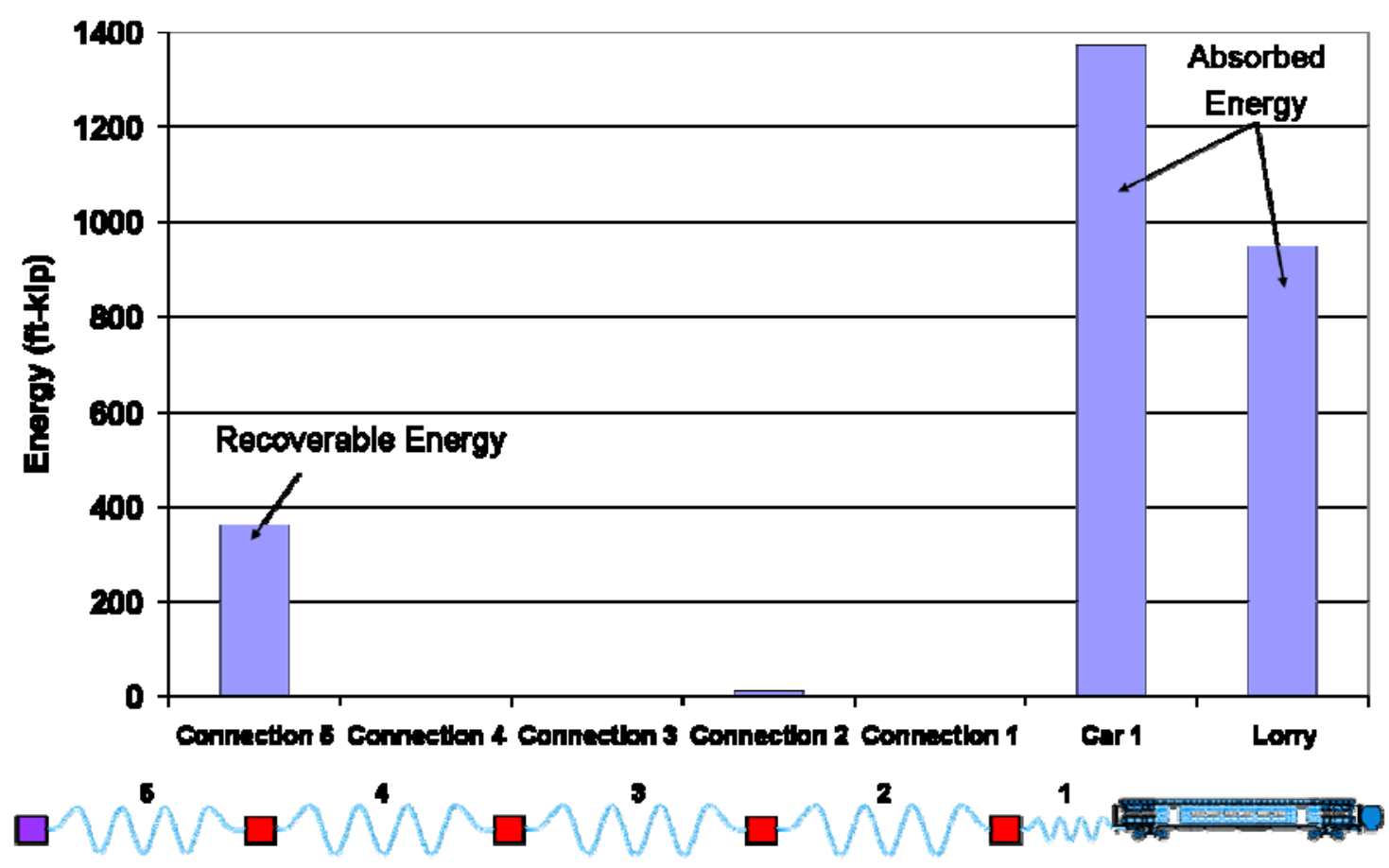

Figure 19. Strain Energy Absorbed by the Consist and Lorry

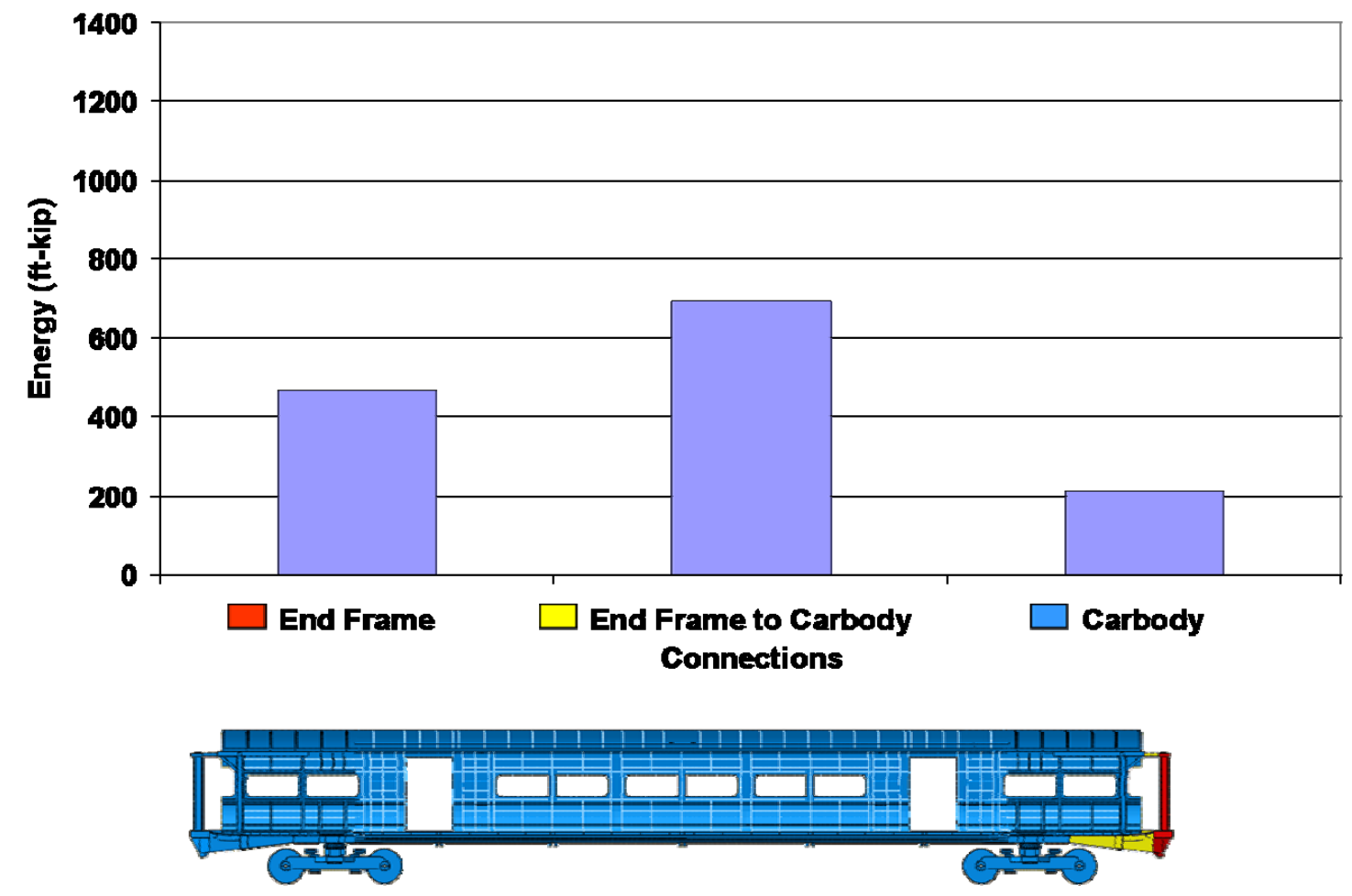

Figure 20. Strain Energy Absorbed by Car 1 


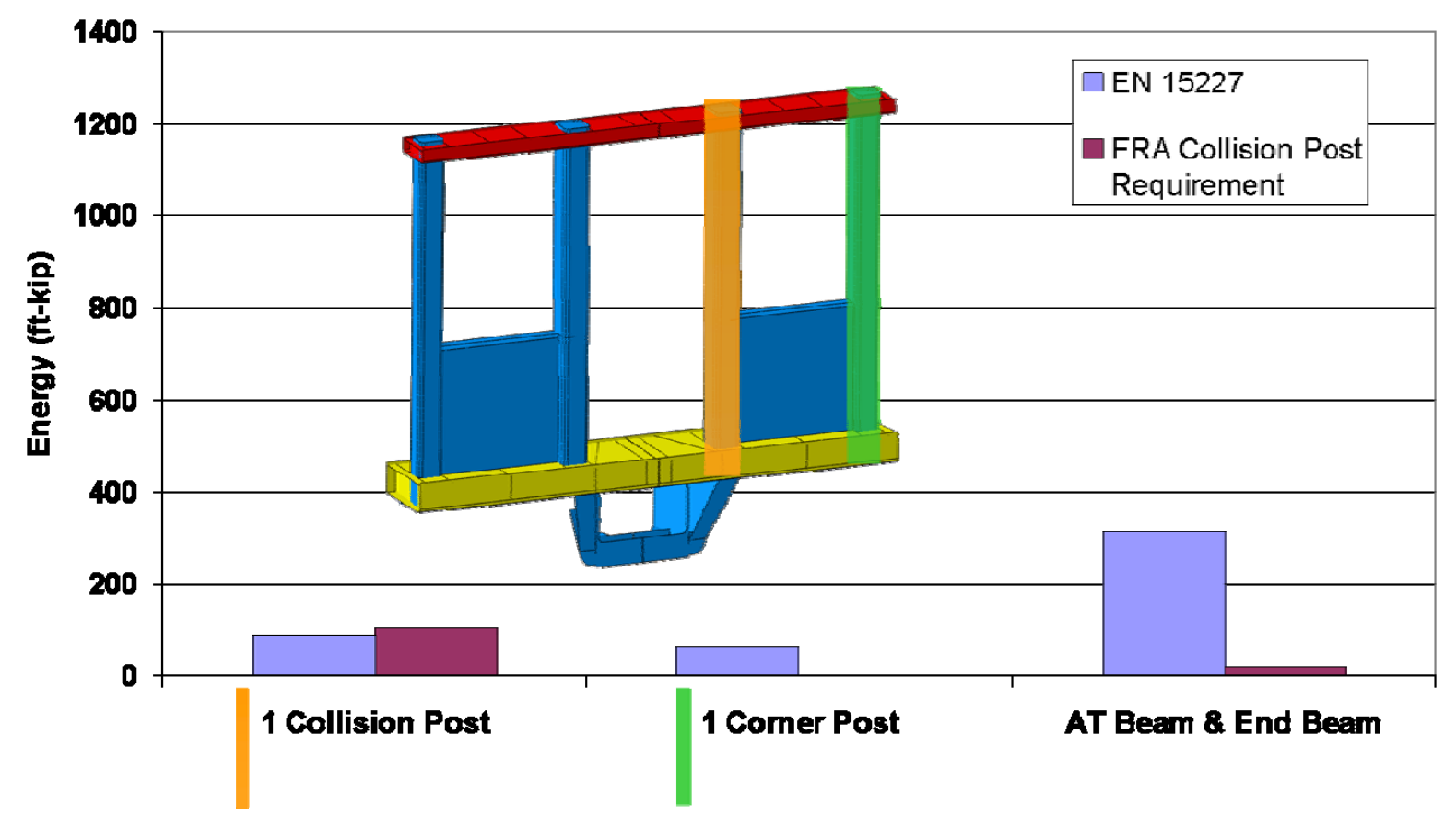

Figure 21. Strain Energy Absorbed by the End Frame

Figure 22 shows the amount of space left in the cab after the impact. Because an M1 Budd cab car with an SOA end frame does not exist in service, the location of the operator's seat in a New Jersey Path train cab was used, as this information was most readily available. Figure 22 shows there are approximately 18 in of space left in front of the operator's seat after the impact. This exceeds the requirement of $300 \mathrm{~mm}$ (11.8 inches). The mean decelerations of the impact were approximately $0.8 \mathrm{~g}$, which is well below the requirement of $7.5 \mathrm{~g}$.

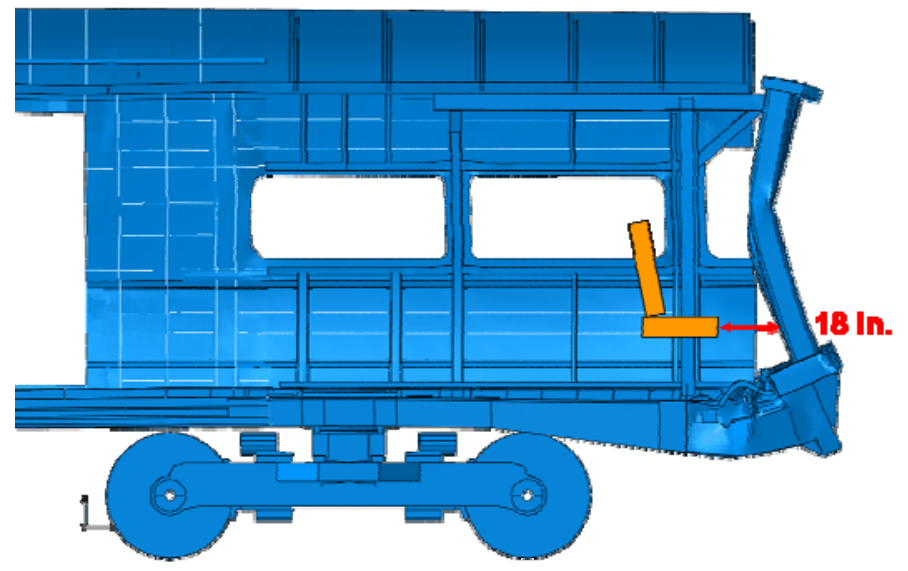

Figure 22. Cab Survival Space
Although the SOA end frame on the M1 Budd cab car is not a CEM car, the EN 15227 standard requirements have been met. The survival space and structural integrity of the occupied areas have been maintained, and the mean decelerations have been kept below $7.5 \mathrm{~g}$.

\section{COMPARISON OF PERFORMANCE STANDARDS}

To make a comparison of these two standards, Table 1 provides a few key crashworthiness parameters. The FRA scenario involves only one car, whereas the EN 15227 scenario involves a complete consist or train unit. The difference in weights of one car, 80 kips, versus a complete consist, 767 kips, is an order of magnitude. The FRA scenario involves the 14-kip impact object moving $19 \mathrm{mph}$, resulting in an initial kinetic energy of $170 \mathrm{ft}$-kip, whereas the EN 15227 scenario involves the 767-kip consist moving $53 \mathrm{mph}$, resulting in an initial kinetic energy of 72,000 ft-kip. The difference in the amount of initial kinetic energy involved is 2 orders of magnitude between the two scenarios. Similarly, the impacting objects are quite different. The FRA scenario involves a rigid impact object, whereas the EN 15227 scenario involves a deformable impact object. In the FRA scenario, this results in the energy being mostly absorbed by the impacted collision post, with no energy absorbed by the impact object. Whereas, in the EN 15227 scenario, both the first car and the impact object absorb large amounts of energy, with very little absorbed by one collision post. 
Table 1. Comparison of FRA Standard and EN 15227

\begin{tabular}{|c|c|c|}
\hline PARAMETER & FRA STANDARD & $\begin{array}{c}\text { EN } 15227 \\
\text { SPECIFICATION }\end{array}$ \\
\hline Type of train & Single car: 80 kips & $\begin{array}{c}\text { Complete train unit: } \\
767 \text { kips }\end{array}$ \\
\hline Impact object & Rigid cart: 14 kips & $\begin{array}{c}\text { Deformable lorry: } \\
33 \text { kips }\end{array}$ \\
\hline Impact speed & $19 \mathrm{mph}$ (cart) & $53 \mathrm{mph}$ (consist) \\
\hline $\begin{array}{l}\text { Initial kinetic } \\
\text { energy }\end{array}$ & 170 ft-kip & 72,000 ft-kip \\
\hline $\begin{array}{l}\text { Energy } \\
\text { absorbed }\end{array}$ & $\begin{array}{c}\text { End frame: } \\
138 \text { ft-kip } \\
\text { Cart: } 0 \\
\text { Collision post: } \\
105 \text { ft-kip }\end{array}$ & $\begin{array}{c}\text { Car 1: } \\
1370 \text { ft-kip } \\
\text { Lorry: } 950 \text { ft-kip } \\
\text { Collision post: } \\
89 \text { ft-kip }\end{array}$ \\
\hline Pass/fail criteria & $\begin{array}{c}\text { Intrusion }<=10 \text { in., } \\
\text { no separation }\end{array}$ & $\begin{array}{l}\text { Operator seat space } \\
>=11.8 \text { in., mean } \\
\text { deceleration }<7.5 \mathrm{~g}\end{array}$ \\
\hline
\end{tabular}

The key parameters of these two scenarios, although they are both grade-crossing collision scenarios involving rail vehicles with impact objects, are very different. Also, comparing the complexity of the analyses conducted for each scenario, the FRA scenario is simpler to analyze, with fewer vehicles required in the analysis, at a lower initial kinetic energy with less deformation, and deformation resulting only in the car and not the impact object. Therefore, the FRA scenario provides clearer metrics of success.

\section{CONCLUSIONS}

The following conclusions can be drawn about the FRA grade-crossing performance standard:

1. It concentrates load on a single post, above the underframe,

2. It can be validated by either analysis or test, and

3. It can be applied to both CEM and non-CEM equipment.

The following conclusions can be drawn about the EN 15227 grade-crossing specification:

1. It distributes the load across the entire end structure,

2. It imparts a significant amount of load in the underframe and roof structure,

3. It can only be validated by analysis, and

4. It assumes CEM equipment.

The FRA scenario also imparts more energy to the impacted post than the EN 15227 scenario requires. The FRA scenario is shown to be easier to analyze as well as easier to test than the EN 15227 scenario. Additionally, the FRA scenario is safer to test because of the levels of initial energy involved. The FRA scenario also provides clearer metrics of success. The FRAcompliant car utilized in the analyses and test conducted for this paper passed both FRA and EN 15227 grade-crossing collision scenarios according to the requirements for each respective standard. However, the analyses show that despite both scenarios providing for energy absorption in a gradecrossing collision, because the manner in which the car is loaded and deformed (concentrated vs. distributed) is different, the FRA performance standard and EN 15227 grade-crossing collision scenarios are not equivalent and mutual compliance is not guaranteed.

\section{ACKNOWLEDGMENTS}

The research performed in this paper was conducted as part of the Equipment Safety Research Program sponsored by the Office of Research \& Development of FRA. Eloy Martinez, Program Manager, led this effort. Luis Maal, FRA Resident Manager at the Transportation Technology Center, managed the full-scale dynamic test effort.

Richard Stringfellow and Christopher Paetsch, of TIAX, ran the FEA for the FRA collision scenario, and monitored the purchase and fabrication of the parts of the SOA end frame, under contract to the Volpe Center. Mark White of Transportation Technology Center, Inc. (TTCI), led the TTCI team that implemented the dynamic test. Tom Roderick of TTCI, led the fabrication and assembly of the SOA end frame and retrofit onto the Budd M1 car. Long Island Railroad donated the Budd M1 car used in the dynamic test.

\section{REFERENCES}

[1] Starlinger, A., Castelli, B., and Gmur, A., "On the Crashworthiness Performance of the New GTW DMU Developed for Capital Metro (Austin, Texas),” American Society of Mechanical Engineers, Paper No. RTDF200874018, September, 2008.

[2] National Transportation Research Board. "Collision of Northern Indiana Commuter Transportation District Train 102 with a Tractor-Trailer, Portage, Indiana, June 18, 1998,” 07/26/1999, RAR-99-03.

[3] 49 CFR Part 238, Notice of Proposed Rulemaking, Passenger Equipment Safety Standards; Front End Strength of Cab Cars and Multiple Unit Locomotives. Washington, DC: U.S. Department of Transportation, Federal Railroad Administration.

[4] EN 15227, Railway Applications-Crashworthiness Requirements for Railway Vehicle Bodies, Ref. No. prEN 15227:2007:E.

[5] Mayville, R. Stringfellow, R., and Martinez, E., "Development of Conventional Passenger Cab Car End Structure Designs for Full Scale Testing,” U.S. Department of Transportation, DOT/FRA/ORD-06/20, December, 2006.

[6] Stringfellow, R., Paetsch, C., and Amar, G., "Development and Fabrication of State-of-the-Art End Structures for Budd M1 Cars," American Society of Mechanical Engineers, Paper No. RTDF2008-74033, September, 2008.

[7] Priante, M., Llana, P., Jacobsen, K., Tyrell, D., Perlman, B., "A Dynamic Test of a Collision Post of a State-of-theArt End Frame Design,” American Society of Mechanical Engineers, Paper No. RTDF2008-74020, September, 2008. 